\title{
Experimental Study on Image-based Position-Force Adaptive Visual Servoing for Constrained Robots under Jacobian and Dynamic Friction Uncertainties*
}

\author{
E.C. Dean-León, L.G. García, V. Parra-Vega \\ Mechatronics Division, CINVESTAV-IPN \\ IPN 2508,Sn P. Zacatenco, México, D.F.,7300. México \\ \{edean, lgarcia,vparra\}@cinvestav.mx
}

\author{
A. Espinosa-Romero \\ FMat, Universidad Autónoma de Yucatán \\ Mérida, Yucatán. México \\ eromero@tunku.uady.mx
}

\begin{abstract}
In this paper an image-based control is introduced to produce simultaneous convergence of the constrained visual position and contact force between the end-effector and the constraint surface. Camera, robot and jacobian parameters are considered uncertain. This new approach is based on a new formulation of the orthogonalization principle used in force control, coined here visual orthogonalization principle. This allows, under the framework of passivity, to yield a synergetic scheme that fuses camera, encoder and force sensor signals. However, when complex friction arises, visual servoing suffers to drive the robot to the desired trajectories, in particular in slow motion and velocity reversals, which are typical motions in this sort of control schemes. Moreover, dynamic friction is usually neglected in motion control and it is not the exception in visual servoing literature. In our case, we explore an interesting result to compensate for uncertain dynamic friction. In order to prove the theory described in this article, the real-time OS, LinuxRTAI, is used to obtain experimental results of this controller on a direct-drive robot manipulator equipped with six axis JR3 force sensor. Results suggest its excellent performance.

Index Terms - Visual Servoing, Adaptive Force Control, Sensor Fusion, Unknown Jacobian, Dynamic Friction.
\end{abstract}

\section{INTRODUCTION}

Robot tasks that involve joint encoders, force sensors, CCD cameras, proximity sensors, haptic interfaces, and tactual devices pose a challenging problem in robotics due to the multisensor nature of the problem. However, it is well known that multisensor-based robot control approaches may offer a solution to very important and relevant, but complex, problems in robotics. In order to achieve sensor fusionbased controller, a careful analysis of the dynamics, sensors behavior, and tasks are required. Furthermore, since physical parameters are in practice uncertain, robustness to parametric uncertainties, are an integral part of the control problem.

\section{A. Motivation}

The task under study is that the robot end effector tracks a visual trajectory along the surface of an object, and at the

${ }^{*}$ This work is supported by CONACyT under doctoral scholarship $\# 158973$, \#158613, and project \#39727-Y same time, control the applied force exerted in the surface by the end-effector, see Figure (1). This problem has been elusive because it is not evident how to deal with vision and force signals, despite lot of the availability of schemes of vision or force. This task is very relevant in many robotic applications. However, for any practical impact, uncertainties must be considered.

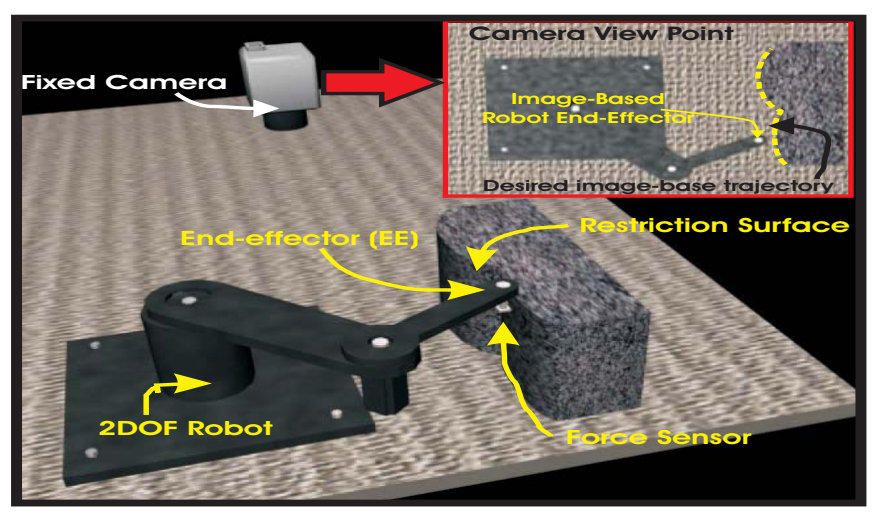

Fig. 1. Robot Force-Vision Experimental System.

On the other hand, joint friction is quite important to compensate because it is a dominant dynamical force in slow and velocity reversal regimes. Therefore, we consider the LuGre model ${ }^{1}$, which reproduces pre-sliding regime at very small displacements and hard nonlinearities for slow motion and velocity reversals, which is typical motion regime of contact tasks and visual servoing ${ }^{2}$. Interestingly, a friction compensator that depends on image errors is derived. The problem stated above is studied in this paper, and a formal solution is provided.

\footnotetext{
${ }^{1}$ This dynamic friction is responsible for limit cycles.

${ }^{2}$ This motion regimen is presented in visual control scheme due to physical restrictions of camera systems.
} 


\section{B. Contribution}

An adaptive controller driven by image errors and contact force errors is proposed to solve by first time the problem possed above. The underlying reason that allows to obtain this result is that a new image-based error manifold is introduced to propose a visual-based orthogonalized principle. Thus, similar results to the case of nonvisual-based orthogonalized principle are obtained. The closed-loop system guarantees exponential tracking of position and force trajectories subject to parametric uncertainties. This scheme delivers a smooth controller and presents formal stability proofs. Moreover, this article shows the experimental validation of the theoretical framework presented, on a two degree-of-freedom directdrive manipulator, provided with a six axis force sensor, interacting with a highly rigid surface. The control system is running over Linux-RTAI operating system. We further extend our proposal to include visual compensation of dynamic friction. Surprisingly the control structure is quite simple, in contrast, the proof is rather involved, though straightforward.

\section{BACKGROUND}

Hybrid vision/force control approaches have been reported [1], [2], [3], and none of them shows robustness to uncertainties, on robot parameters and camera parameters. In a different path, the authors Xiao et al. [4], present an interesting scheme of hybrid vision force control in an uncalibrated environment, but their approach does not deal with uncertainties of robot parameters, and exhibits a very complex control law. The authors Hosoda et al. [13], solves a part of the problem with an adaptive control for visual and force tracking, where the proposed controller has on-line estimators to deal with the camera and environment uncertainties, however, this scheme needs the explicit knowledge of the manipulate kinematics that, in this work, has been assumed as unknown. With respect to force control, Arimoto solved by first time the simultaneous control of position and force using the full nonlinear dynamics subject to parametric uncertainties without coordinate partitioning. This was possible through judicious design of extended error, that is based on the orthogonalization principle. Afterwards, several schemes have been proposed based on the orthogonalization principle, however these schemes have not been extended or combined beyond constrained robots.

\section{NONLINEAR ROBOT DYNAMICS}

\section{A. Constrained robot dynamics}

The constrained robot dynamics arises when its end effector is in contact to infinitely rigid surface. Considering the generalized position $q \in \Re^{n}$ and velocity coordinates $\dot{q} \in \Re^{n}$, this system can be modeled as a set of differential algebraic equation as follows [5]

$$
\begin{aligned}
H(q) \ddot{q}+C(q, \dot{q}) \dot{q}+g(q) & =\tau+J_{\varphi+}^{T}(q) \lambda-F(\dot{q}, \dot{z}, z) \\
\varphi(q) & =0
\end{aligned}
$$

where matrix $H(q) \in \Re^{n \times n}$ stands for the symmetric positive definite manipulator inertia matrix; $C(q, \dot{q}) \dot{q} \in \Re^{n}$ stands for the vector of centripetal and Coriolis torques; $g(q) \in \Re^{n}$ is the vector of gravitational torques, $F(\dot{q}, \dot{z}, z)$ is the dynamic friction $^{3}, J_{\varphi+}(q)=\frac{J_{\varphi}}{J_{\varphi} J_{\varphi}^{T}}$ is the constrained normalized jacobian of the the kinematic constraint $\varphi(q)=0$ or rigid surface assumed frictionless with continuous gradient and $\lambda$ stands for the constrained lagrangian, or contact force, finally $\tau \in \Re^{n}$ stands for the vector of input torque control.

Adding and subtracting to (1)-(2) the following linear parametrization $H(q) \ddot{q}_{r}+C(q, \dot{q}) \dot{q}_{r}+g(q)=y_{r} \theta_{b}$, where the known regressor $y_{r}=y_{r}\left(q, \dot{q}, \dot{q}_{r}, \ddot{q}_{r}\right) \in \Re^{n \times p}$ and the unknown constant vector $\theta_{b} \in \Re^{p}, p>0$, produces the open loop error equation

$$
H(q) \dot{S}_{q}=\tau+J_{\varphi+}^{T}(q) \lambda-C(q, \dot{q}) S_{q}-y_{r} \theta_{b}
$$

with joint error surface $S_{q}$ defined as

$$
S_{q}=\dot{q}-\dot{q}_{r}
$$

where $\dot{q}_{r}$ stands for the nominal reference of joint velocities, not yet defined.

\section{CAMERA MODEL}

The static pin hole camera model is used, considering thin lens without aberration [6]. To introduce the camera model, first consider the robot direct kinematics

$$
x_{b}=f(q)
$$

where $x_{b} \in \Re^{n}$ represents the position of robot end effector in cartesian space, $q \in \Re^{n}$ is the vector of generalized joint displacements, and $f(\cdot): \Re^{n} \rightarrow \Re^{n}$. Then, the differential kinematics of robot manipulator, which relates velocities in cartesian space $\dot{x}_{b} \in \Re^{n}$ to joint space velocities $\dot{q} \in \Re^{n}$, is defined as follows

$$
\dot{x}_{b}=J(q) \dot{q}
$$

Now, the visual position $x_{s} \in \Re^{2}$ of robot end effector in image space (screen) is given by [6]

$$
x_{s}=\alpha R(\theta) x_{b}+\beta
$$

where $\alpha$ is the scale factor ${ }^{4}, R(\theta) \in S O(3)$, and $\beta \in \Re^{2}$ that depends on intrinsic and extrinsic parameters of camera ${ }^{5}$. The differential camera model is then

$$
\dot{x}_{s}=\alpha R(\theta) \dot{x}_{b}
$$

${ }^{3}$ For a clear exposition, firstly, $F(\dot{q}, \dot{z}, z)$ will be considered zero, however in Section VIII it will be treated.

${ }^{4}$ Without loss of generality, $\alpha$ can be considered as a scalar matrix $2 \times 2$.

${ }^{5}$ Focal distance, depth of field, translation of camera center to image center, distance between optical axe to the robot base. 
where $\dot{x}_{s} \in \Re^{2}$ determines the visual robot end effector velocity. Notice that the constant transformation $\alpha R(\theta)$ maps statically robot cartesian velocities $\dot{x}_{b}$ into visual velocities or visual flow $\dot{x}_{s}$.

Using equation (5) (7), equation (8) becomes

$$
\dot{x}_{s}=\alpha R(\theta) J(q) \dot{q}
$$

Thus, the inverse differential kinematics for robot manipulator in terms of visual velocities ${ }^{6}$ becomes

$$
\dot{q}=J(q)^{-1} R(\theta)^{-1} \alpha^{-1} \dot{x}_{s} \Rightarrow \dot{q}=J_{R i n v} \dot{x}_{s}
$$

This relation is useful to design the nominal reference of joint velocities $\dot{q}_{r}$ in the following section.

\section{Visual ORThogonalization Principle}

Since $\varphi(q)=0 \forall t$, then its time derivative yields $\frac{d}{d t} \varphi(q)=\frac{\partial \varphi(q)}{\partial q} \frac{d q}{d t} \equiv J_{\varphi}(q) \dot{q}=0$. This means that $J_{\varphi}(q)$ is orthogonal to $\dot{q}$. That is, $\dot{q}$ belongs to the orthogonal projection matrix $Q$ of $J_{\varphi}(q)$ [5] as:

$$
Q=I-J_{\varphi+}^{T}(q) J_{\varphi}(q)
$$

As we can see, $Q$ spans the tangent plane at the contact point, therefore, $J_{\varphi}$ and $Q$ are orthogonal complements. In other words, if the robot end effector is in contact with the constraint surface, then $Q \dot{q}=\dot{q} \rightarrow Q Q \dot{q}=Q \dot{q} \equiv \dot{q}$, that is, $Q$ is idempotent; therefore naturally, $Q J_{\varphi}^{T}=0$.

These properties are fundamental to establish the visual orthogonalization principle as follows.

Firstly consider $\dot{q}_{r}$ in terms of two orthogonal nominal references of velocity $\dot{q}_{s}$ and force $\dot{q}_{f}$, as follows

$$
\dot{q}_{r}=\dot{q}_{s}+\dot{q}_{f}
$$

Notice that, we are also interested in designing an image based servo visual force control without computing inverse kinematics $^{7}$, then nominal reference $\dot{q}_{r}$ must be designed in terms of nominal visual reference and nominal force reference as follows

$$
\dot{q}_{r}=Q J_{R i n v} \dot{x}_{r}+\beta J_{\varphi}^{T} \dot{q}_{r f}
$$

with $\dot{q}_{s}=Q J_{R i n v} \dot{x}_{r}$ and $\dot{q}_{f}=\beta J_{\varphi}^{T} \dot{q}_{r f}$. The visual and force nominal references, $\dot{x}_{r}, \dot{q}_{r f}$, respectively, are defined as follows:

Consider the next nominal visual reference of velocities

$\dot{x}_{r}=\dot{x}_{s d}-\alpha \Delta x_{s}+S_{s d}-\gamma_{s 1} \int_{t_{0}}^{t} S_{s \delta}-\gamma_{s 2} \int_{t_{0}}^{t} \operatorname{sign}\left(S_{s \delta}\right)$

${ }^{6}$ With $J_{R i n v} \in \Re^{n \times n}$ whose entries are functions of robot and camera parameters.

${ }^{7}$ to eliminate the inverse kinematics calculus and to reduce the control law computational cost. This is also one byproduct of this scheme. where $\dot{x}_{s d}$ stands for desired visual velocity trajectory, and $\Delta x_{s}=x_{s}-x_{s d}$ is the visual position error. With the visual error surface defined as:

$$
S_{s \delta}=S_{s}-S_{s d} \equiv\left(\Delta \dot{x}_{s}+\alpha \Delta x_{s}\right)-S_{s}\left(t_{0}\right) e^{-\kappa_{s} t}
$$

where $\Delta \dot{x}_{s}=\dot{x}_{s}-\dot{x}_{s d}$ defines visual velocity error, $\kappa_{s}>0$ and $\gamma_{s_{i}}=\gamma_{s_{i}}^{T} \in \Re_{+}^{n \times n}, i=1,2$.

Now, let consider the nominal force reference as

$$
\dot{q}_{r f}=\Delta F-S_{d F}+\gamma_{F 1} \int_{t_{0}}^{t} S_{F \delta}+\gamma_{F 2} \int_{t_{0}}^{t} \operatorname{sign}\left(S_{F \delta}\right)
$$

with the force error surface determined as:

$$
S_{F \delta}=S_{F}-S_{F d} \equiv \Delta F-S_{F}\left(t_{0}\right) e^{-\kappa_{F} t}
$$

where $\Delta F=\int_{t_{0}}^{t} \Delta \lambda(\zeta) d \zeta, \Delta \lambda=\lambda-\lambda_{d}, \lambda_{d}$ is the desired contact force, $\kappa_{F}>0$, and $\gamma_{F_{i}}=\gamma_{F_{i}} \in \Re_{+}^{n \times n}, i=1,2$.

Using equations (10) (16) into (4), the visual orthogonalized joint error surface arise as follows

$$
\begin{aligned}
S_{q} & =\dot{q}-\dot{q}_{r} \equiv Q \dot{q}-\dot{q}_{r} \\
& =Q J_{R i n v} \dot{x}_{s}-Q J_{R i n v} \dot{x}_{r}-\beta J_{\varphi}^{T} \dot{q}_{r f} \\
& =Q J_{R i n v} S_{v s}-\beta J_{\varphi}^{T} S_{v F}
\end{aligned}
$$

with

$$
\begin{gathered}
S_{v s}=S_{s \delta}+\gamma_{s 1} \int_{t_{0}}^{t} S_{s \delta}+\gamma_{s 2} \int_{t_{0}}^{t} \operatorname{sign}\left(S_{s \delta}\right) \\
S_{v F}=S_{F \delta}+\gamma_{F 1} \int_{t_{0}}^{t} S_{F \delta}+\gamma_{F 2} \int_{t_{0}}^{t} \operatorname{sign}\left(S_{F \delta}\right)
\end{gathered}
$$

where $S_{v s}$ stands for the visual error manifold and $S_{v F}$ stands for the force error manifold.

Notice that $S_{q}$ is composed of two orthogonal complements $Q J_{R i n v} S_{v s}$ depending on image coordinate errors, and $\beta J_{\varphi}^{T} S_{v F}$ depending of integral of contact force errors. Thus, tracking errors $\Delta x_{s}$ and $\Delta F$ can be controlled independently, since they are mapped to orthogonal complements.

Remark 1. The above definition assumes exact knowledge of $J_{\text {Rinv }}$. However, in practice, it stands as a very restricted assumption. Therefore, we need to design a uncertain manifold $S_{q}$ taking into consideration the uncertainty of $J_{\text {Rinv }}$. To this end, consider

$$
\widehat{\dot{q}}_{r}=Q \widehat{J_{\text {Rinv }}} \dot{x}_{r}+\beta J_{\varphi}^{T} \dot{q}_{r f}
$$

with $\widehat{J_{\text {Rinv }}}$ an estimated of $J_{\text {Rinv }}$, such that rank $\widehat{J^{-1}(q)}$ and $\widehat{R_{\alpha}^{-1}(\theta)}$ are full rank $\forall q \in \Omega$, where the robot workspace free of singularities is defined by $\Omega=$ $\left\{q \mid \operatorname{rank}(J(q))=n, \forall q \in \Re^{n}\right\}$, and $\forall \theta \in \Re$. Thus, substituting (19) into (4), we have the uncalibrated joint error surface

$$
\begin{aligned}
\hat{S}_{q} & =\dot{q}-\widehat{\dot{q}}_{r} \\
& =Q J_{\text {Rinv }} \dot{x}_{s}-Q \widehat{J_{R i n v}} \dot{x}_{r}-\beta J_{\varphi}^{T} \dot{q}_{r f}
\end{aligned}
$$


where $\hat{S}_{q}$ is available because $\dot{q}$ and $\widehat{\dot{q}}_{r}$ are available. Adding and subtracting $Q J_{R i n v} \dot{x}_{r}$ to (20) we obtain

$$
\begin{aligned}
\hat{S}_{q} & =Q J_{R i n v} S_{v s}-\beta J_{\varphi}^{T} S_{v F}-Q \Delta J_{R i n v} \dot{x}_{r} \\
& =S_{q}-Q \Delta J_{R i n v} \dot{x}_{r}
\end{aligned}
$$

where $\Delta J_{R i n v}=\widehat{J_{R i n v}}-J_{R i n v}$.

\section{OPEN LOOP ERROR EQUATION}

Using (19), the uncertain parametrization $y_{r} \theta_{b}$ becomes

$$
H(q) \widehat{\ddot{q}_{r}}+C(q, \dot{q}) \hat{\dot{q}}_{r}+g(q)=y_{r} \hat{\theta}_{b}
$$

which introduces discontinuous terms. To avoid introducing high frequency discontinuous signals, continuous and discontinuous signals are separated as in [14]. Therefore (22) becomes

$H(q) \widehat{\ddot{q}_{r}}+C(q, \dot{q}) \widehat{\dot{q}_{r}}+g(q)=y_{\text {cont }} \hat{\theta}_{b}+H\left(Q \gamma_{s 2} z_{s}-\beta J_{\varphi}^{T} \gamma_{F 2} z_{f}\right)$

Adding and subtracting (23) to (1)-(2), we obtain finally the open loop error in function of $\left(q, \dot{q}, \hat{\dot{q}}_{r}, \hat{\ddot{q}}_{r c o n t}\right)$ as follows:

$$
\begin{aligned}
H(q) \widehat{\dot{S}_{q}}= & \tau-C(q, \dot{q}) \widehat{S_{q}}+J_{\varphi+}^{T}(q) \lambda- \\
& y_{\text {cont }} \hat{\theta}_{b}+H\left(Q \gamma_{s 2} z_{s}-\beta J_{\varphi}^{T} \gamma_{F 2} z_{f}\right)
\end{aligned}
$$

Now the main result is presented.

\section{Control Design}

Theorem 1 Assume that initial conditions and desired trajectories belong to $\Omega$, and consider the robot dynamics (1)(2) in closed loop with the following visual adaptive forceposition control law

$$
\begin{aligned}
\tau= & -K_{d} \hat{S}_{q}+y_{\text {cont }} \hat{\theta}_{b}+J_{\varphi+}^{T}(q)\left[-\lambda_{d}+\eta \Delta F\right]+ \\
& \beta J_{\varphi}^{T}(q) *\left[\tanh \left(v_{F} S_{F \delta}\right)+\eta \int_{t_{o}}^{t} \operatorname{sgn}\left(S_{F \delta}\right)\right] \\
\dot{\hat{\theta}}_{b}=- & -\Gamma y_{\text {cont }}^{T} \hat{S}_{q}
\end{aligned}
$$

where $\Gamma \in \Re_{+}^{p \times p_{+}}, K_{d} \in \Re_{+}^{n \times n}, \beta, \eta>0$. If $K_{d}$ is large enough and error of initial conditions are small enough, and if $\gamma_{s 2} \geq\left\|\frac{d}{d t}\left\{R_{\alpha}(\theta) J(q)\left[\hat{S}_{q}+\left(\Delta J_{\text {Rinv }}\right) \dot{x}_{r}\right]\right\}\right\|, \gamma_{F 2} \geq$ $\left\|\frac{d}{d t}\left[\left(J_{\varphi} J_{\varphi}^{T}(q)\right)^{-1} J_{\varphi} \hat{S}_{q}\right]\right\|$, then exponential convergence of visual and force tracking errors is guaranteed.

Proof: A detailed proof of the theorem can be found in [14].

Remark 2. Since the continuous $\tanh (*)$ is substituted instead of sign $(*)$, upper bounds $\varepsilon_{2}$ and $\varepsilon_{3}$ are greater. To induce the second order sliding mode, and therefore exponential convergence of tracking errors, it suffices to tune $\gamma_{s 2}$ and $\gamma_{F 2}$ to a larger value. If sign $(*)$ would have been used, then smaller $\gamma_{s 2}$ and $\gamma_{F 2}$ would have been tuned, but at the price of chattering on the control input.

\section{DYNAMIC FRICTION COMPENSATION}

The following LuGre [7] dynamic friction model is considered

$$
\begin{aligned}
F(\dot{q}, \dot{z}, z) & =\sigma_{0} z+\sigma_{1} \dot{z}+\sigma_{2} \dot{q} \\
\dot{z} & =-\sigma_{0} h(\dot{q}) z+\dot{q} \\
h(\dot{q}) & =\frac{|\dot{q}|}{\alpha_{0}+\alpha_{1} \exp ^{-\left(\dot{q} / \dot{q}_{s}\right)^{2}}}
\end{aligned}
$$

where matrix parameters $\sigma_{0}, \sigma_{1}, \sigma_{2}$ are diagonal definite matrices $n \times n$, the state $z \in \Re^{n}$ stands for the position of the bristles, $\alpha_{0}, \alpha_{1}>0$, and $\dot{q}_{s}>0$. This model exhibits the following complex dynamic friction effects (see [7] for more details). These effects involve a very complex dynamics around the trivial equilibrium, for bidirectional motion, and for very small displacements, the forces that comes out from this model makes impossible to reach the origin due to the limit cycles induced and the potentially unstable behavior.

Substituting (27) into (1)-(2) yields

$H(q) \ddot{q}+C(q, \dot{q}) \dot{q}+\sigma_{12} \dot{q}+g(q)+\sigma_{0} z-\sigma_{01} h(\dot{q}) z=\tau+J_{\varphi_{+}}^{T}(q) \lambda$

where $\sigma_{01}=\sigma_{0} \sigma_{1}$ and $\sigma_{12}=\sigma_{1}+\sigma_{2}$. Substituting the uncalibrated nominal reference (19) in (28), just like (22), lies the next equation

$H(q) \widehat{\ddot{q}}+C(q, \dot{q}) \widehat{\dot{q}}+\sigma_{12} \widehat{\dot{q}}+g(q)+\sigma_{0} z-\sigma_{01} \hat{h}(\dot{q}) z=\tau+J_{\varphi+}^{T}(q) \lambda$

Similar to [8], only the part of the equation (29) that is linear in parameters (LP) is rewritten in terms of the uncalibrated nominal reference $\left(\widehat{\dot{q}_{r}}, \widehat{\ddot{q}_{r}}\right)^{T} \in \Re^{2 n}$ as follows

$$
H(q) \hat{\ddot{q}}_{r}+C(q, \dot{q}) \hat{\dot{q}}_{r}+\sigma_{12} \hat{\dot{q}}_{r}+g(q)=Y_{r} \hat{\Theta}_{b}+\sigma_{12} \hat{\dot{q}}_{r}
$$

Notice that $y_{r} \hat{\theta}_{b} \neq Y_{r} \hat{\Theta}_{b}$. To be able to cast the problem of non-LP of equation (27) as a disturbance rejection problem, [8] proposes a discontinuous virtual regressor, which in turn yields chattering, with harmful consequences to real physical systems. To avoid chattering the following virtual continuous regressor is introduced

$$
\frac{\sigma_{01} \alpha_{01}}{\alpha_{0}}|\dot{q}| \tanh \left(\xi_{f} \hat{S}_{q}\right)+\sigma_{0} \alpha_{01} \tanh \left(\xi_{f} \hat{S}_{q}\right)=Y_{f} \Theta_{f},
$$

where $\alpha_{01}=\alpha_{0}+\alpha_{1}, \tanh (q)$ is the continuous hyperbolic tangent function, and $\xi_{f}>0$. If we add and subtract (30) and (31) to (28), the following parametrization arises

$$
H(q) \hat{\dot{S}}_{q}+C(q, \dot{q}) \hat{S}_{q}+\sigma_{12} \hat{S}_{q}=\tau-\mathcal{F}-Y \hat{\Theta}+J_{\varphi+}^{T}(q) \lambda
$$

with

$$
\begin{aligned}
\mathcal{F}= & \sigma_{0}\left\{z+\alpha_{01} \tanh \left(\xi_{f} \hat{S}_{q}\right)\right. \\
& +\alpha_{0}{ }^{-1} \sigma_{1} \alpha_{01}|\dot{q}| \tanh \left(\xi_{f} \hat{S}_{q}\right) \\
& \left.-\sigma_{1}|\dot{q}| z\left(\alpha_{0}+\alpha_{1} \exp ^{-\left(\dot{q} / \dot{q}_{s}\right)^{2}}\right)^{-1}\right\}
\end{aligned}
$$


where $Y=\left[Y_{r}, Y_{f}\right]$, and $\hat{\Theta}=\left[\hat{\Theta}_{b}^{T}, \Theta_{f}^{T}\right]^{T}$. Finally, solving (32) for $H(q) \widehat{\dot{S}}_{q}$, yields the following open-loop visual error dynamics subject to dynamic friction

$$
H(q) \widehat{\dot{S}}_{q}=-C(q, \dot{q}) \widehat{S}_{q}-\sigma_{12} \widehat{S}_{q}+\tau-\mathcal{F}-Y \hat{\Theta}+J_{\varphi+}^{T}(q) \lambda
$$

Finally, consider the following visual adaptive force-position control law

$$
\begin{aligned}
\tau= & -K_{d} \hat{S}_{q}+Y \hat{\Theta}+J_{\varphi+}^{T}(q)\left[-\lambda_{d}+\eta \Delta F\right]+ \\
& \beta J_{\varphi+}^{T}(q) *\left[\tanh \left(v_{F} S_{F \delta}\right)+\eta \int_{t_{0}}^{t} \operatorname{sgn}\left(S_{F \delta}\right)\right] \\
\hat{\Theta}= & -\Gamma Y^{T} \hat{S}_{q}
\end{aligned}
$$

where $\Gamma \in \Re^{p \times p_{+}}, K_{d} \in \Re_{+}^{n \times n}, \beta, \eta>0$. Now, the following result is presented.

Theorem 2 Assume that initial conditions and desired trajectories belong to $\Omega$, and consider the controller (35)-(36). If $K_{d}$ is large enough and a error of initial conditions are small enough, and if $\gamma_{s 2} \geq\left\|\frac{d}{d t}\left\{R_{\alpha}(\theta) J(q)\left[\hat{S}_{q}+\Delta J_{\text {Rinv }} \dot{x}_{r}\right]\right\}\right\|, \quad \gamma_{F 2} \geq$ $\left\|\frac{d}{d t}\left[\left(-\beta J_{\varphi} J_{\varphi}^{T}(q)\right)^{-1} J_{\varphi} \hat{S}_{q}\right]\right\|$, then exponential convergence of visual and force tracking errors is guaranteed.

Proof: A detailed proof of the theorem can be found in [14].

\section{EXPERIMENTAL STATION}

Robot parameters and constant gains used in the simulations are: $\left(m_{1}, m_{2}\right)=(6.72,2.03) \mathrm{Kg},\left(l_{1}, l_{2}\right)=$ $(0.4,0.3) m$ and $\Gamma=1, \kappa_{f}=20, \gamma_{f}=3.0, \eta=0.029, \beta=$ $1.0, K_{d}=90, \alpha=40, \kappa s=20, \gamma_{s}=7.8$. Friction parameters: $\sigma_{0}=30000, \sigma_{1,2}=2, \alpha_{0,1}=(4,0.4), \dot{q}_{s}=0.01$. An image of the experimental setup is depicted in Fig. 2.

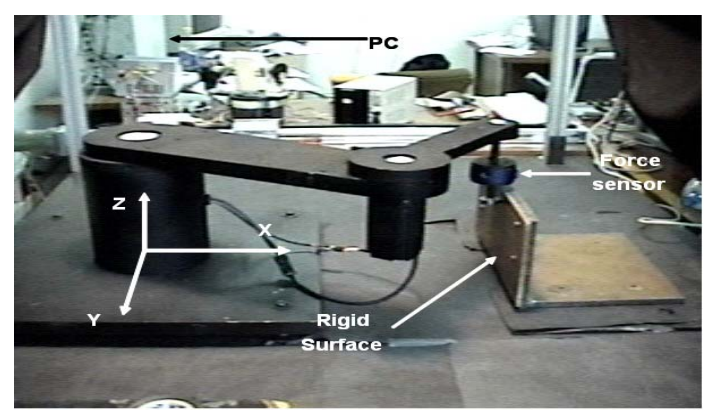

Fig. 2. Experimental setup.

\section{A. The Hardware}

A SONY DFW-VL500 Firewire CCD digital camera is placed with its optical axis tangent to the robot work space, and its visual area covering completely the reachable space of the robot arm. Direct-drive Yaskawa AC servomotors SGM08A314 and SGM-04U3B4L with 2048 pulse encoders are directly coupled to the links of the 2-dof arm. Digital drive electronics from the Yaskawa servopacks (SGD-08AS and SGDA-04AS) are integrated. A six-axis force-moment sensor 67M25A-I40-200N12 by JR3 Inc., provided with a DSP Based Interface System for PCI bus, is mounted at the end effector of the robot. The sensor has a maximum rating of $\pm 200 N$ in the $\mathrm{XY}$ axes and twice in the $\mathrm{Z}$ axis. The tool used in the experiments is a rigid aluminum probe with a bearing in its tip, implanted to reduce contact friction, as shown in Fig. 2. The robot task is to move its tool-tip along a specified trajectory over the steel surface while at the same time exerts a specified profile of force normal to the surface.

\section{B. The firmware and software}

The control system is running on a PC over Linux-RTAI operating system, with two real-time parallel processes. The control process at a sampling rate of $1 \mathrm{KHz}$ and the visual acquisition process at sampling rate of $33 \mathrm{~Hz}$. Low level programming provides communication with the $\mathrm{CCD}$ digital camera via IEEE-1394 protocol. Also, offers the interface to a Sensoray 626 I/O card which contains internal quadrature encoder interface, 14 bit resolution analog outputs and digital I/O. Velocity is computed using a dirty Euler numerical differentiation formula filtered with a lowpass second order Butterworth filter, with a cutoff frequency of $20 \mathrm{~Hz}$.

\section{Results}

The robot is initialized with a high gain PD since the parametric uncertainty is $100 \%$. The inertial frame of the whole system is at the base of the robot and the contact surface is at $y=136$ pixel rendering a $X Z$ plane. The experiment is performed as follows:

1) From $t=(0,3) s$, free motion of the end-effector until makes contact with surface. The end effector lasts 2 more seconds static.

2) From time $t=(5,8) \mathrm{s}$ the tool-tip exerts a desired profile of force $5 \mathrm{~N}$, while moving from $(230,136)$ pixels to $(299,136)$ pixels

3) From $t=(8,12) s$ the exerted force is incremented 7.5 $\mathrm{N}$, while moving from $(299,136)$ pixels to $(230,136)$ pixels, as can be seen in Fig. 3 A) and Fig. 4 B), Fig. 4 C), \& Fig. 4 D).

Fig. 3 B) shows the input torques. It can be observed that there are not saturation and smooth behavior. Fig. 4 A) depicts the real and desired trajectories in the cartesian plane.

\section{CONClusions}

This paper introduces a novel scheme for adaptive image based visual servoing/force control in a constrained dynamical system. The main feature is the ability to fuse image coordinates into a orthogonal complement of joint velocities, 
and contact forces in the orthogonal complement of integral of contact forces. Using this, exponential convergence arises for image-based position-velocity and contact forces even when robot parameters, camera parameters and analytical jacobian are considered unknown. Additionally, it is proposed a image-based compensator of uncertain dynamic friction, which is usually neglected in visual servoing, but it is of particularly concern in visual motion tasks, because the motion regime is slow, with velocity reversals. Experimental results validate the predicted theoretical performance. Notice that the system stability remains even when the robot end-effector motion switches from free motion to constrained motion due to its passivity properties, under a set of conditions [12]. Experimental results comply dully to the theoretical stability properties.

\section{REFERENCES}

[1] A. Namiki, Y. Nakabo, I. I. M. I., High speed grasping using visual and force feedback. Proceedings of the 1999 IEEE International Conference on Robotics and Automation, Detroit, MI, pages 3195-3200.

[2] J. Baeten, W. Verdonck, H. B. J. D. S., Combining force control and visual servoing for planar contour following. Machine Intelligence and Robotic Control. 2000, 2(2):69-75.

[3] S. Jorg, J. Langwald, J. S. G. H. C. N., Flexible robot assambly using a multi-sensory approach, Procc. of the 2000 IEEE Int. Conf. Robot. and Autom., San Francisco, CA, pages 3687-3694.

[4] Xiao, D., Sensor-hybrid Position/Force control of a robot manipulator in an un calibrated enviroment, IEEE Trans. Control Systm Technology, 2000, 8(4):635-645.

[5] S. Arimoto, Y. H. Liu and T. Naniwa, Model Based Adaptive Hybrid Control for Geometrically Constrained Robot Manipulator, Proc. IEEE Int. Conf. on Rob. and Autom. 1996, pp. 618-623.

[6] S. Hutchinson, G. Hager, P. C., A tutorial on visual servo control, Trans. on Robotics and Automation. 1996. Vol. 6, 651-670

[7] Canudas de Wit, C., Olsson, H., and Astrom, K.J., A New Model for Control of Systems with Friction, IEEE Transactions of Automatic Control, 1995, Vol. 40., No. 3, 419-425.

[8] Panteley, E., Ortega, R., \& Gafvert, M., An Adaptive Friction Compensator for Global Tracking in Robot Manipulators, Systems and Control Letters, 1998, Vol. 33, No. 5, 307 - 313.

[9] V. Parra-Vega, S. Arimoto, Y. L. G. H. P. A., Dynamic sliding PID control for tracking of robot manipulators: Theory and experiments, IEEE Transactions on Robotics and Automation. 2003. Vol. 19., 967976.

[10] Parra-Vega, V., Adaptive compensation of dynamic friction in finite time of 1 DOF mechanical system. American Control Conference, 2001. Proceedings of the 2001 ,Volume: 3 , 25-27 June 2001, pp: 2462 - 2467 vol. 3

[11] García-Valdovinos, L. G., Seguimiento Perfecto en Tiempo Finito de Robots Manipuladores Sujetos a Fricción Dinámica, Master Thesis, Dept of EE, Mechatronics Division, CINVESTAV-IPN, México, D.F., 2003

[12] P. Akella, V. Parra-Vega., Arimoto S. and T. Tanie, Discontinuous Adaptive Control for Robot Manipulators Executing Free and Constrained Tasks, Proc. of the IEEE Robotics and Automation, San Diego (1994), pages 3000-3007.

[13] Hosoda, K., Igarashi, K., Asada, M., Adaptive Hybrid Control for Visual and Force Servoing in an Unknown Environment, IEEE Robotics \& Automation Magazine, December 1998, pp 39-43.

[14] Dean-León,E., García-Valdovinos, L., Parra-Vega, V., EspinosaRomero, A., Uncalibrated Image-based Position ForceAdaptive Visual Servoing for Constrained Robots under Dynamic Friction Uncertainties, IEEE/RSJ International Conference on Intelligent Robots and Systems, IROS 2005. August 2-6, Edmonton, Alberta, Canada.

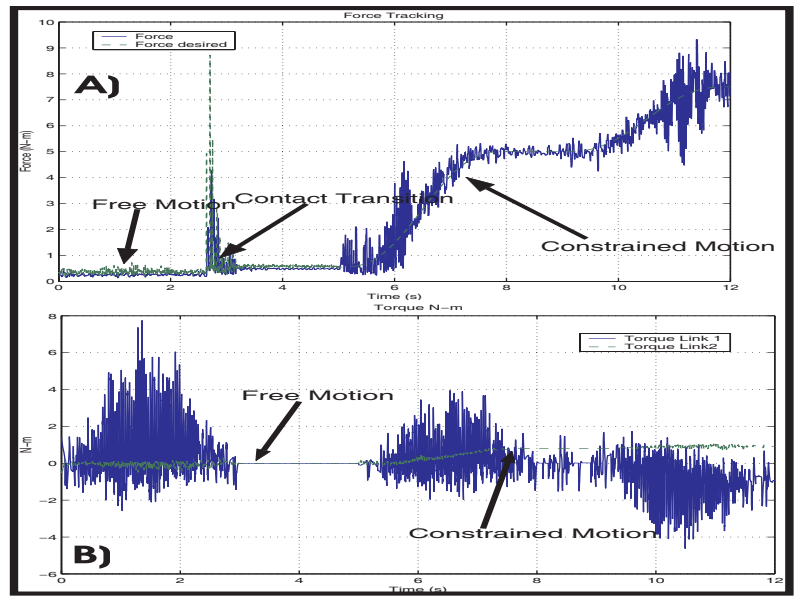

Fig. 3. A)Force Tracking. B)Smooth input torques.

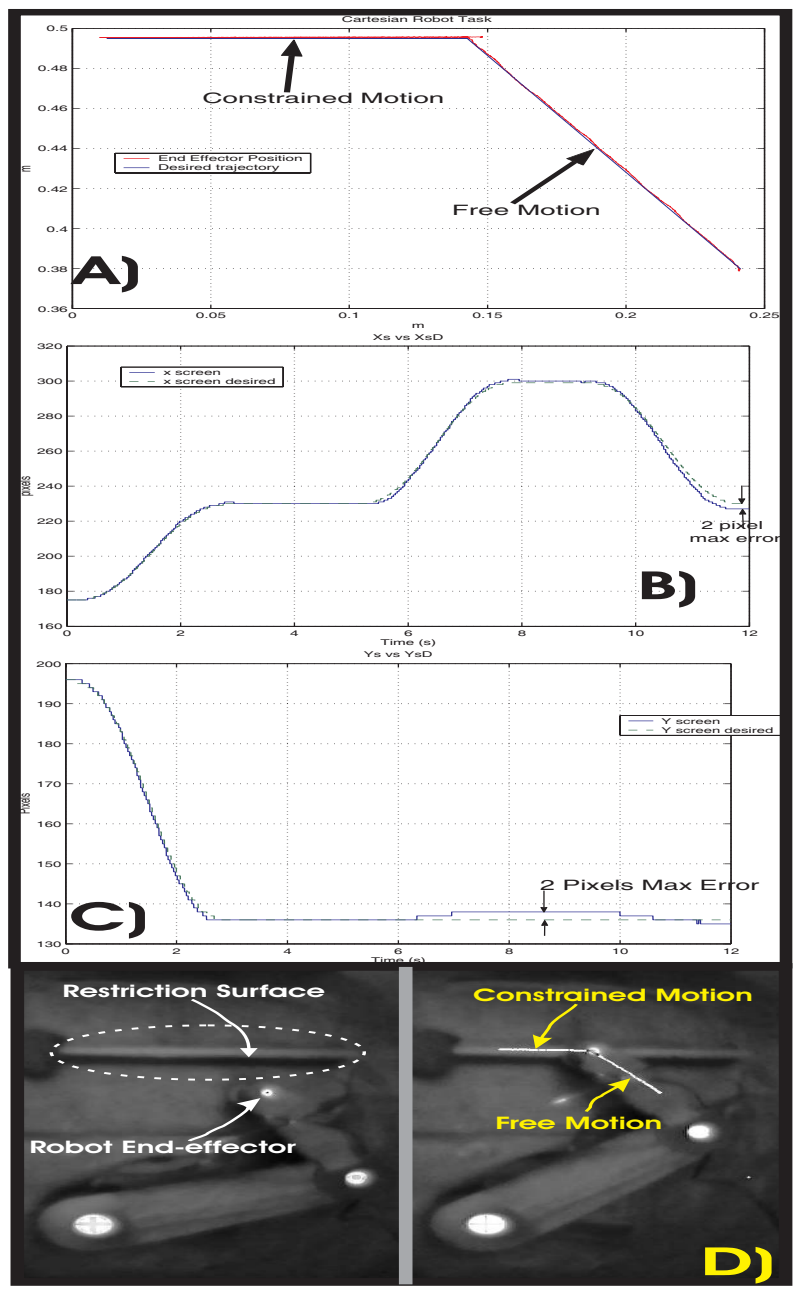

Fig. 4. A)Cartesian robot task in robot space (m). B)End effector $\mathrm{x}$ position in image space (pixels). C)End effector y position in image space (pixels). D)Experiment camera view point. 